\section{Molecular Transport Junctions: An Introduction}

\section{Cherie R. Kagan and Mark A. Ratner, Guest Editors}

\begin{abstract}
This issue of MRS Bulletin on molecular transport junctions highlights the current experimental and theoretical understanding of molecular charge transport and its extension to the rapidly growing areas of molecular and carbon nanotube electronics. This introduction will outline the progress that has been made in understanding the mechanisms of molecular junction transport and the challenges and future directions in exploring charge transport on the molecular scale. In spite of the substantial challenges, molecular charge transport is of great interest for its intrinsic importance to potential single-molecule electronic, thin-film electronic, and optoelectronic applications.
\end{abstract}

Keywords: carbon nanotubes, charge transport, molecular electronics, molecular junctions, optoelectronics, self-assembly.

\section{Molecular Electronics: Definition and History}

Molecular electronics is most simply defined as electronics whose behavior is dictated by the chemical, physical, and electronic structures of molecules. This definition is very broad; it includes conductive polymers and even the insulating polymer layers on metal wiring. In common use, molecular electronics refers to molecular structures whose characteristic features are on the nanoscale and that contain between one and a few thousand molecules. The simplest challenge involves determining the structure/function relationships for electronic transport (intra- or intermolecular) through a junction containing one or a few molecules as the transport medium and with either two (source/drain) or three (source/gate/drain) electrodes. Such transport structures are a dominant theme of contemporary molecular electronics and are the focus of this issue of MRS Bulletin.

The history of molecular electronics is brief. Some of the earliest work was performed at the laboratory of Hans Kuhn, working with Mann, Polymeropoulos, and Sagiv. ${ }^{1}$ Using organic adlayers on solid substrates, this group reported some of the earliest (1971) reproducible electrical transport measurements through organic molecules. They also developed some of the first effective self-assembly techniques for preparing structures in which molecules adhere to surfaces not by simple dispersion forces, but by molecular bond formation.

Following Kuhn's work, there were a number of important follow-up measurements. ${ }^{2}$ In addition, there were some visionary (if perhaps premature) papers ${ }^{3}$ suggesting the use of single molecules as rectifiers and extensive device ideas involving molecular logic structures. ${ }^{4}$

The great step forward came in 1983, with the development of scanning probe microscopy (SPM). ${ }^{5}$ The capability to both manipulate and measure molecular structures topologically and spectroscopically prompted a renewed focus on the possibilities of molecular electronics. Continuing advances in synthesizing organic molecules with possible device applicability, and in their assembly and measurement, have led to increasing interest in the field and to substantial advances in our understanding. ${ }^{6}$

Starting roughly a decade after the invention of SPM, applications to molecular structures and their transport behavior ap- peared. Important early work included the use of $\mathrm{C}_{60}$ as an electromechanical gate device, ${ }^{7}$ transport measurements on organic molecules with thiol end groups assembled between a gold surface and gold nanocrystals, ${ }^{8}$ and the introduction of break junction ${ }^{9}$ measurements to characterize currents in one (or a few) molecules.

Theoretical ideas for interpreting the current/voltage characteristics in molecular junctions began to appear in the 1990s. Some early approaches used the Landauer formulation, ${ }^{10}$ originally developed for semiconductor devices. In this simple picture, transport through molecular junctions is interpreted in terms of elastic scattering, and the conductance is given as the product of the quantized unit of conductance $(12.9 \mathrm{k} \Omega)^{-1}$ and a transmission coefficient describing how effective a molecule is in scattering the incoming electron from the upstream lead into the downstream lead. More powerful formulations, including the nonequilibrium Green's function (NEGF) approach, were also introduced in the mid-1990s. ${ }^{11}$ Integrating a proper description of the molecule with an appropriate description of the leads is the challenge that these formulations face; the NEGF seems capable of including elastic and inelastic effects as well as vibronic (vibrational electron) coupling and system/bath interaction.

In this issue of MRS Bulletin, leading researchers provide a quick tour of some of the thematic areas of interest. In the first article, Hersam and Reifenberger discuss charge transport through molecular junctions and provide a brief description of how to measure and interpret such transport behavior.

Ghosh et al. provide a theoretical overview of molecular charge transport structures. They introduce the different theoretical formulations, and provide guidance both for building understanding and interpreting experiments.

In the third article, Kushmerick et al. discuss conductance with different test beds and inelastic tunneling measurements.

In the last article, Avouris provides an introduction to carbon nanotube junctions, structures, transport, and devices.

\section{Synthesis, Interpretation, and Device Aspects}

In order to understand molecular structure/function relationships, it is necessary to synthesize appropriate molecules, fabricate (incorporate) them into a certain structure (junctions), and probe their behavior. Questions of structure and stability are crucial.

Both nature and synthetic chemistry have exploited the unique bonding character of 
the carbon atom and a range of metal coordination environments to prepare a vast number of molecular systems. Routine bench-scale chemical synthesis produces $10^{23}$ molecules at a time, and each molecule is identical. Chemical functionalization of the molecules and van der Waals, $\pi-\pi$, covalent, and hydrogen bonding forces enable self-assembly of single molecules and molecular monolayers on surfaces. The diversity of molecular systems suggests the use of molecular materials in a wide range of applications (due to their structural and optical properties and processability) in packaging, mechanical, dye, passive and active optical, and resist technologies, and promises applications (arising from their electronic properties) in electrical and optoelectronic devices.

Carbon nanotubes (CNTs), discovered by Iijima in 1991, are frequently classified as "molecular" systems since their electronic structure is discrete. CNTs possess remarkable mechanical, electrical, optoelectronic, and thermal properties, as detailed in the following paragraphs. They are also thermally stable, making them compatible with Si processing and therefore particularly exciting for potential electronic and optoelectronic devices.

Understanding the correlation between molecular junction structure and electrical properties is of fundamental and technological interest. Fabricating molecular junctions less than $\sim 10 \mathrm{~nm}$ in size presents a tremendous challenge and has made it difficult to compare reported measurements with theoretical work carried out to describe current flow in molecular junctions (see article in this issue by Ghosh et al.). Because CNTs can be much longer than small molecules, junctions with lengths ranging from the nanometer to the millimeter scales can be fabricated by standard lithographic techniques, and device structures can be studied to provide feedback between experimental and theoretical work. Since molecules and CNTs have many similarities, theoretical modeling of current flow in both molecule- and CNTbased junctions follows a generalized form of the Landauer theory. Insight into the electrical characteristics of molecular and CNT junctions and the influence of structure, conformation, interfaces with substrates or contacts, and environment is the first step in the pursuit of molecular-scale and CNT devices and is important to the improvement of thin-film organic electronic and optoelectronic devices.

The chemical diversity of molecular systems and the ability to synthetically tailor molecular structure has generated interest in molecular analogues of insulators, interconnects (wires), rectifiers, switches (transis- tors), memory elements, and sensors. Similarly, metallic and semiconducting CNTs are exciting as potential interconnects, diodes, switches, memory elements, and sensors. More recently, molecular-scale and CNT electronic devices have received enormous attention, driven by the anticipation that conventional Si technology is running ever faster toward its scaling limits as the channel length of transistors has shrunk to the nanometer $(<100 \mathrm{~nm})$ size regime. Molecular-scale devices are expected to represent the ultimate in device scaling, as molecular lengths can be as small as $\sim 1 \mathrm{~nm}$, although limitations of tunneling and electrostatics, as in Si devices, set a lower bound on device dimensions (for the example of a molecular transistor, $\sim 3 \mathrm{~nm}$ ).

The simplicity of solution- or gas-phase self-assembly of molecular systems offers an opportunity for low-cost fabrication. Functionality that can be chemically tailored may provide for complexity in molecular devices that is not found in Si devices. For example, while scaling in $\mathrm{Si}$ devices is governed by pushing device dimensions ever smaller, in molecular devices it may be better to make larger molecules to achieve functionality and performance not found in Si devices. In CNT field-effect transistors (CNTFETs), the ballistic transport at small dimensions, reduced dimensionality, and the lack of dangling bonds that provides flexibility in interface/device design have led to promising demonstrations of device performance (see the article by Avouris in this issue).

Charge transport in molecular junctions is also important to the progress of thin-film organic devices such as light-emitting diodes (LEDs), photovoltaics (PVs), thin-film transistors (TFTs), and memory cells. The metal-molecule and metal-semiconductor junctions in each of these applications significantly limit the performance of thin-film molecular devices. Organic LEDs (OLEDs) have recently been commercialized in car radio displays and indicator lights and are being developed by many manufacturers for large-area displays. In OLEDs, charge injection across the molecule-metal interface requires high-voltage and, therefore, high-power operation and limits the stability and lifetime of devices. In organic and organic-inorganic PVs, recombination losses at metal-molecule and metal-semiconductor interfaces represent one of the biggest challenges to device efficiency. Similarly, in organic TFTs the moleculecontact interface presents electronic barriers to charge injection that give rise to current crowding at low voltage, measured as sublinear $I-V$ characteristics in the triode region, and that necessitate higher device operating voltages.

\section{Structure/Function Relationships}

Molecular electronics entails at least two entities, the molecule and the electrode. From an energetic viewpoint, the molecular states are discrete molecular orbitals, while the electrodes (metallic or semiconducting) consist of bands characterized by continuous densities of states. The mix of discrete and continuous structures represents much of the challenge in understanding molecular electronic transport.

As indicated earlier, formulating the transport through the discrete structure requires a molecular Hamiltonian description and a Green's function description of the interaction of that molecule with the macroscopic leads.

When voltage is placed across the molecule, the electronic structure of the molecular species is no longer in equilibrium, although it can be in a steady state. The molecule tries to adjust to an electrochemical potential of one magnitude at the cathode side and another magnitude at the anode side; this causes some polarization within the molecule, which acts effectively as a polarized capacitor.

One of the reasons that CNTs are the most advanced areas of molecular electronics is that the community has a reasonably good understanding of their structure. Because CNTs can be prepared with lengths far exceeding a few nanometers, measurements can be (and have been, see Figure 1) made of the nature of the electrostatic potential across the CNT, and of the approximate CNT geometries. For junctions containing organic molecules - in particular, the very popular thiol/gold structural motif-we have no direct structural evidence concerning the geometry. This makes calculations and mechanistic deduction quite complex. Indeed, understanding the structure will be crucial if the kinds of structure/function relationships that are of primary interest in molecular electronic junctions are to be compared directly to experiment. Although the structures are generally only crudely understood, there have been some major advances in our understanding of how molecular behavior influences transport in junctions. Highlights of these advances include the following:

1. The comparison between the rate constant (the rate at which electrons would transfer from a donor at one end of a molecular bridge to an acceptor at the other end) and the conductance through that same molecular bridge has been clarified. ${ }^{12}$ This permits interesting aspects of the mechanism - such as the transition between coherent and incoherent transport as a function of either length or temperatureto be compared. 
a

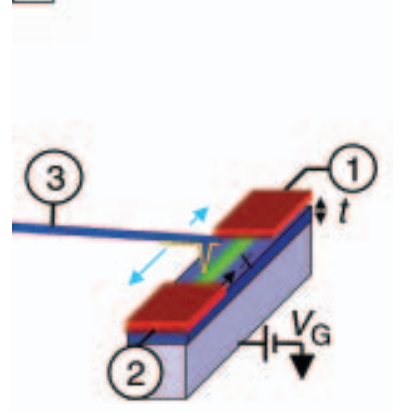

b

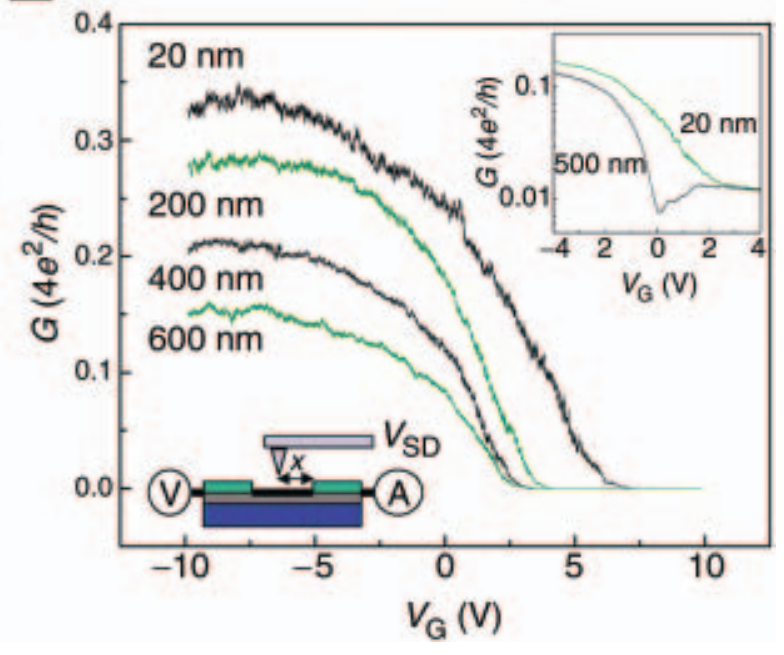

Figure 1. (a) A carbon nanotube transistor (channel length, $1.2 \mu \mathrm{m}$; nanotube diameter, $2 \mathrm{~nm} ; \mathrm{t}=$ gate oxide thickness; $\mathrm{V}_{g}$ is the applied gate voltage). A conductive atomic force microscope (AFM) probe is positioned over the device to study the channel length dependence of the device characteristics and the electrostatics and contacts of the device. Electrodes, marked (1) and (2), and the AFM tip (3) can serve as the voltage source, current probe, or voltage probe in the measurements. (b) The measured channel-length-dependent conductance (in units of the quantum conductance), as a function of $\mathrm{V}_{g}$, where $\mathrm{x}$ is the channel length, $\mathrm{V}_{t i p}=100 \mathrm{mV}$, and $\mathrm{t}=500 \mathrm{~nm} \cdot{ }^{20} \mathrm{~V}_{S D}$ is the source-drain voltage. Similarly (inset), the characteristics of a second device are plotted on a log scale, where $\mathrm{t}=200 \mathrm{~nm}$. (Reprinted with permission from Reference 20.)

2. The importance of covalent interactions at the molecule/metal interface (effectively reducing the Schottky barriers and permitting more control of the molecular levels by the applied voltage) has been clarified. ${ }^{13}$ In nanotubes, true ohmic junctions (for p-type transport) apparently can be prepared $^{14}$ using palladium as the electrode material.

3. The importance of molecular recognition behavior in the construction of junctions and in preparing both adlayers and specific quantum dot junctions is now understood. 4. Dynamical stereochemistry of molecules has been used as the basis for both modeling $^{15}$ and experimental demonstration ${ }^{16}$ of memory and switching applications. This stereochemical variation is one of the unique properties of molecules that most strongly differentiates molecular from more traditional semiconductor electronics.

5. A comparison has been made of transport through junctions containing single molecules with transport through those based on molecular adlayer films; once again, the results highlight the crucial role that contacts play in molecular junction transport. In particular, a substantial reduction in contact resistance has been demonstrated using covalent interactions at carbon electrodes,; ${ }^{17}$ less effective mixing occurs through Lewis interactions at metal/thiol or isocyano links.
6. The importance of the electrostatic potential and its control has been demonstrated clearly, both in measurements and models.

7. Gating due to molecular geometric change is going to be general at ambient temperature, simply because molecules do move at room temperature. Such gating can completely dominate the measured $I-V$ behavior. If controlled, such gating may lead to interesting device applications, ${ }^{15,16,18}$ both in two-electrode and three-electrode configurations.

8. Specific device applications, both in simple molecular transport junctions and in such areas as molecular sensing and molecular optoelectronics, have been widespread and impressive. The great sensitivity of transport through molecular junctions to the electrostatic potential suggests that sensing using transport modification is effective and powerful.

Elegant recent work by $\mathrm{Ho}$ and his collaborators, ${ }^{19}$ discussed in the article by Hersam and Reifenberger, demonstrates the capability of current techniques to assemble junctions on an atom-by-atom basis and then characterize the local densities of states using transport perpendicular to the molecular axis. Extending this understanding to the charge transport behavior, and providing comparably detailed structure/ function relationships, represents perhaps the greatest challenge to our understanding of molecular junctions.

\section{CNT and Molecular Behaviors}

Developing a further understanding of the electronic characteristics and fabrication of molecular- and CNT-based junctions and devices presents unique challenges.

The electronic properties of CNTs have been studied by scanning probe microscopies and optical spectroscopies. Figure 1 shows a CNTFET in which the lithographically defined $1.2 \mu \mathrm{m}$ channel length is large enough for a conductive AFM probe to measure the channel length dependence of the device characteristics, electrostatics, and contacts. ${ }^{20}$ CNTs are being aggressively explored to understand the characteristics of different species of nanotubes, their contact physics, and environmental influences, and to engineer high-quality CNT devices.

Present synthesis routes typically prepare batches of CNTs containing both semiconducting and metallic tubes (determined by their chirality) with a range of diameters. Work has proceeded on functionalizing CNTs with organic molecules in an effort to solubilize them, to separate different species of CNTs, to place the tubes on surfaces, and to electronically manipulate or dope them. While considerable progress has been made in regard to chemical manipulation and synthetic routes, processing to separate and position CNTs presents major challenges to the integration and fabrication of CNT junctions and devices.

In contrast to CNTs, while molecules can be synthesized and self-assembled from solution onto surfaces, the nanometer length scale of molecules makes the study and device fabrication of molecular junctions very challenging. SPM (see Hersam and Reifenberger) offers the resolution and control to characterize single molecules and molecular monolayers on surfaces. SPM has been used to probe the dynamics of molecular conductance on surfaces. Derivatives of 2,5-di(phenylethynyl-4', $4^{\prime \prime}$ thio)benzene (the nitro/amine derivative showed negative differential resistance in vertical structures) ${ }^{21}$ and, more recently, alkanedithiol molecules (Figure 2) ${ }^{22}$ show stochastic switching. Researchers are using SPM to close in on the origin of conductance modulations of molecules on surfaces. Both recent observation of switching in alkanedithiols and computations of the energetics and transport properties of differing junction geometries suggest that the thiol-Au contact may be responsible for the observed fluctuations.

While SPM provides well-controlled junctions and measurements of electron density and density of states, the junctions 
consist of one chemical contact and one tunneling contact, so SPM does not directly measure junction conductance. Mechanically controllable break (MCB) junctions, as shown in Figure $3,{ }^{23}$ have been used ${ }^{9}$ to make two chemical contacts to a single molecule and measure transfer in molecular junctions. MCB junctions are formed using piezoelectrics to push on the fabricated metal structure, controllably breaking the junction, and to vary the separation between the metal tips. The $I-V$ characteristics of some molecular MCB junctions are similar to theoretical calculations and are moleculespecific. The $I-V$ characteristics are stable for many voltage sweeps, but are unstable over longer times, as seen by the different features in the two sets of characteristics collected on the same sample. In effect, the two sets of characteristics represent different molecular junctions, as the junction is broken and remade at the contacts, creating a different spectral density for the molecule in the junction.

Scanning probe microscopy and $\mathrm{MCB}$ junctions cannot be integrated into device geometries. Two-terminal vertical sandwich structures, as shown in Figure 4, were first used to fabricate molecular monolayer junctions and can be fabricated in a crossbar geometry. Monolayer junctions are prepared by assembling the molecules onto a bottom electrode and depositing a top metal electrode, where in principle the monolayer height defines the electrode separation. Molecular monolayer junctions in this geometry have shown low leakage for insulating molecules, rectification in donoracceptor molecules (Figure 4), ${ }^{23}$ and negative differential resistance/switching in some conjugated molecules. The clear drawback of vertical sandwich structures is that the top metal electrode is deposited on the molecules and may partially or completely penetrate or react with the monolayer, depending on the choice of metal. For metalmolecule-metal structures, this often results in junctions with $I-V$ characteristics that are shorted or that have been misinterpreted. For metal-molecule-semiconductor junctions, length-dependent electrical characteristics have been measured for densely packed alkyl monolayers, suggesting that the monolayer coverage is sufficiently dense to mask direct metal-Si pathways. ${ }^{24}$

Concern over top metal deposition and the limitation of providing only two terminal measurements has led to the pursuit of lateral junction geometries. Recently, electromigration has been used ${ }^{25}$ to open up $\sim 1 \mathrm{~nm}$ spacing at the constriction in metal lines, as shown in Figure 5. The semiconductor support provides a third, although distant (relative to the sourcedrain spacing) and therefore weakly cou- pled, gate electrode. Organometallic and organic molecules and small metal clusters have been measured in these junctions. Low-temperature $I-V$ characteristics are highly non-ohmic, consistent with Coulomb blockade (conductance gaps dominated by single-particle charging energy), and Kondo resonance (zero bias conductance, believed to originate from the spin and orbital degrees of freedom in the metal centers). The junctions formed by electromigration or electrochemistry provide a new platform with $\sim 1 \mathrm{~nm}$ gaps for studying the electrical behavior of single smallmolecule systems.
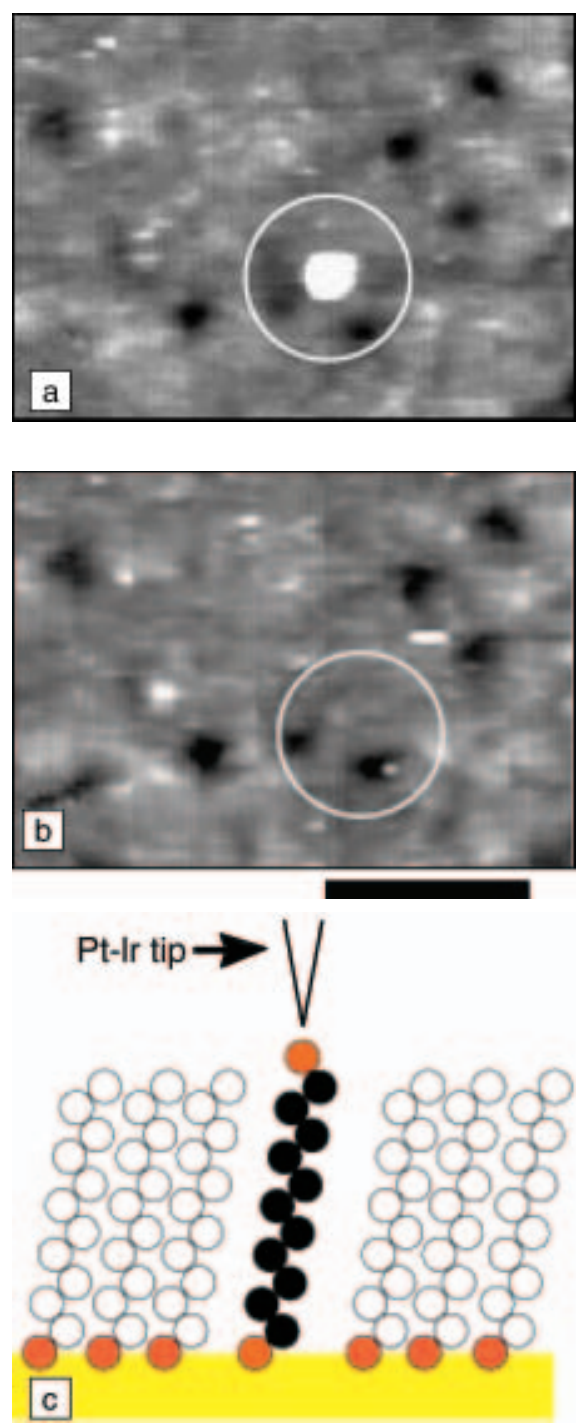

Figure 2. (a), (b) Scanning probe micrographs of alkanedithiols inserted in an alkanethiol monolayer assembled on $\mathrm{Au}$ (c). The size bar is $10 \mathrm{~nm}$. Stochastic switching is observed as the octanedithiols blink on (a) and off (b). (Reprinted with permission from Reference 22.)
Instead of pushing the dimensions of the channels to $\sim 1 \mathrm{~nm}$ separations, recent work $^{26}$ has used e-beam lithography to prepare reproducible junctions and chemistry to bridge the separation between the electrodes, as shown in Figure 6. This
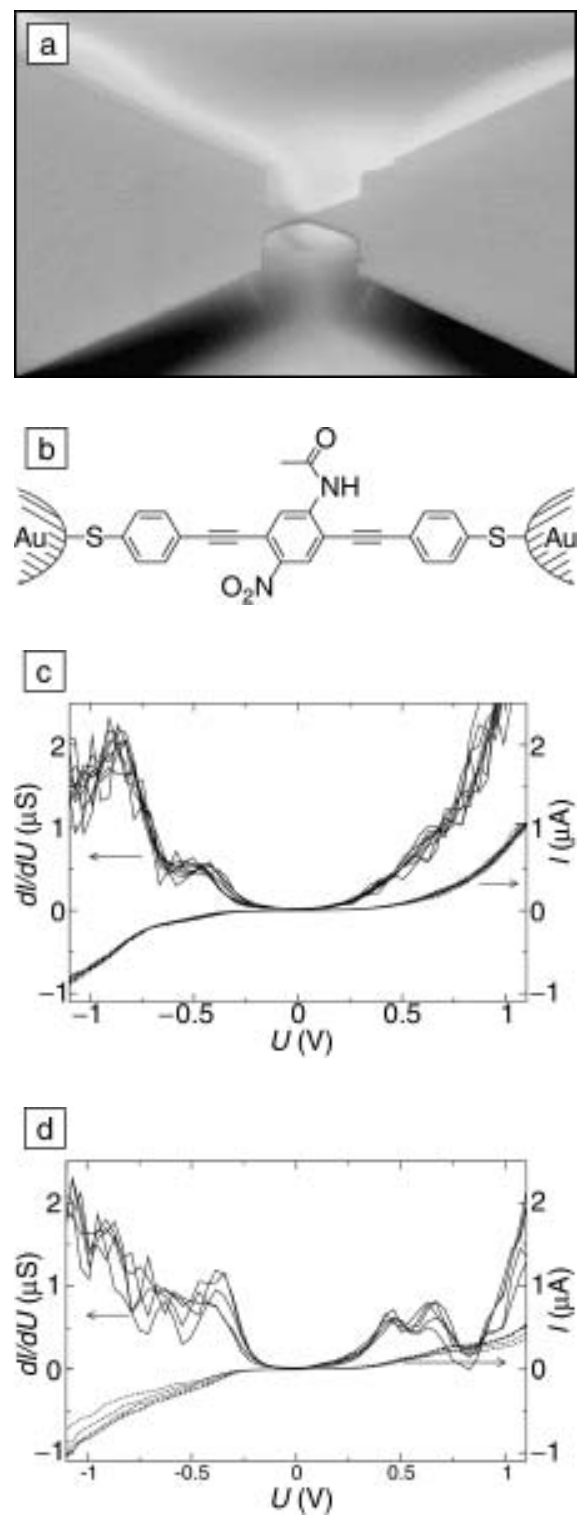

Figure 3. (a) Scanning electron micrograph of a mechanically controllable break junction used to measure the conductance of 1,4-bis(2'-paramercaptophenyl)-ethinyl-2-acetyl-amino5-nitro-benzene. (b) Chemical structure. (c), (d) The I-V characteristics (right axis) and the numerically differentiated $\mathrm{dl} / \mathrm{dU}$ (left axis) values are shown for (c) a junction repeatedly scanned and (d) a subsequent junction after reconfiguration of the molecules between the metal electrodes. (Reprinted with permission from Reference 23.) 

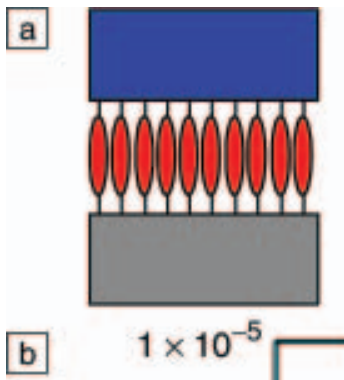

b

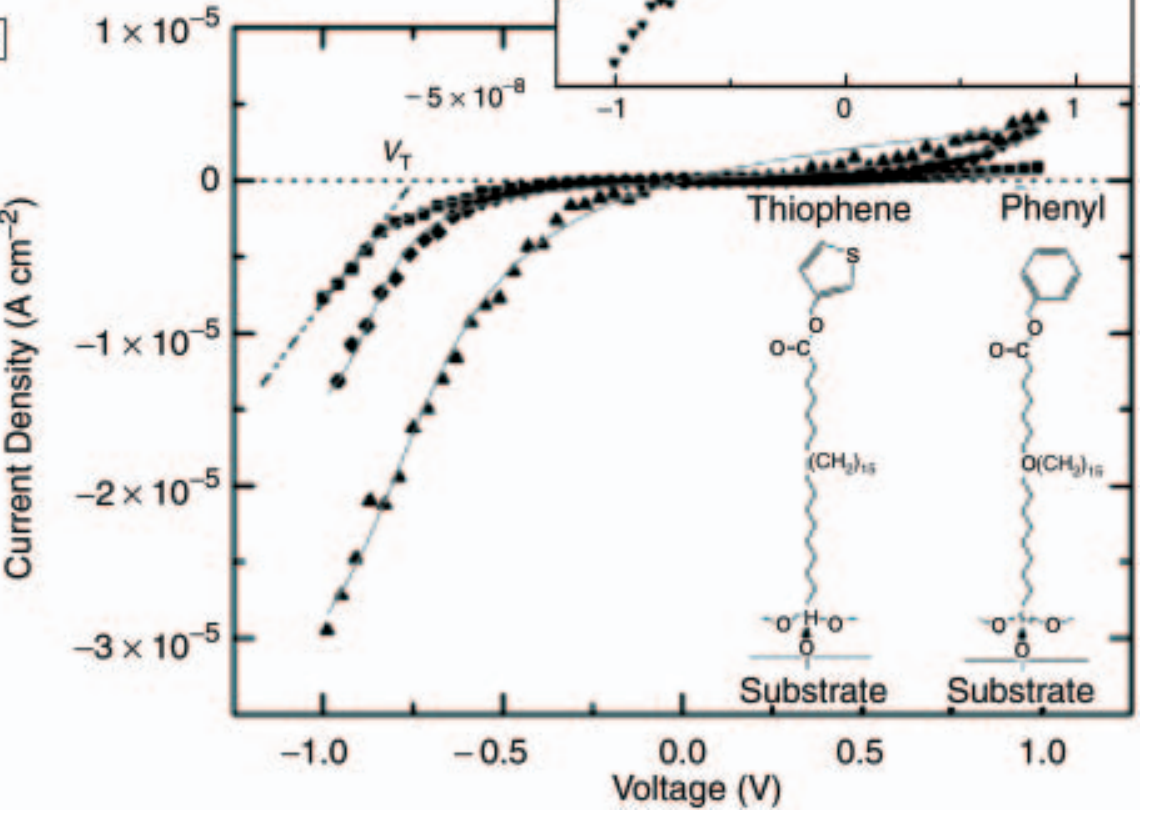

Figure 4. (a) Schematic illustration of a two-terminal vertically fabricated junction. (b) I-V characteristics showing rectification of a thiophene-terminated oct-7-en-1-trichlorosilane self-assembled monolayer (triangles), and phenyl-terminated (squares) and thiophene-terminated (diamonds) heptadec-16-en-1-trichlorosilane self-assembled monolayers, in a molecular junction between $\mathrm{Al}$ and $\mathrm{n}^{+}$Si electrodes. The top graph shows the graphical determination of the threshold voltage. $V_{\mathrm{T}}$ is taken as the intercept of the linear extrapolation of the I-V curve and the zero of the y axis. (Reprinted with permission from Reference 24.) approach also allows a gate to be placed in proximity to the junction, allowing penetration of the gate field in the channel. This approach was demonstrated for metalmetal coordination compounds by templating the assembly of the compounds off the electrodes, using thiol chemistry, and adding molecules layer-by-layer to bridge the channel. $I-V$ characteristics show consistent reversible traces at low voltage and a single, irreversible negative differential resistance at high voltage.

These examples illustrate the many approaches being pursued to understand molecular junctions and highlight some important future directions. Thiol chemistry on Au or carboxylic acids and silanes on $\mathrm{SiO}_{2}$ have been the most heavily studied systems, largely for historical reasons. Many studies suggest that instability in the Au-thiol contact may give rise to observed $I-V$ characteristics, pointing to the importance of controlling the selfassembly using the many functionalities provided by chemistry. Nearly all schemes to create molecular junctions lack information regarding the geometry of the molecule(s) in the junctions, making it almost impossible to correlate the molecule-junction structure with its $I-V$ characteristics. Overcoming this limitation requires new tools to address the molecularscale geometry, as well as continued improvement and new ideas to fabricate reproducible and reliable molecular-scale junctions.

The fields of molecular and carbon nanotube junctions, while presenting different research challenges, are yielding an exciting fundamental understanding of their
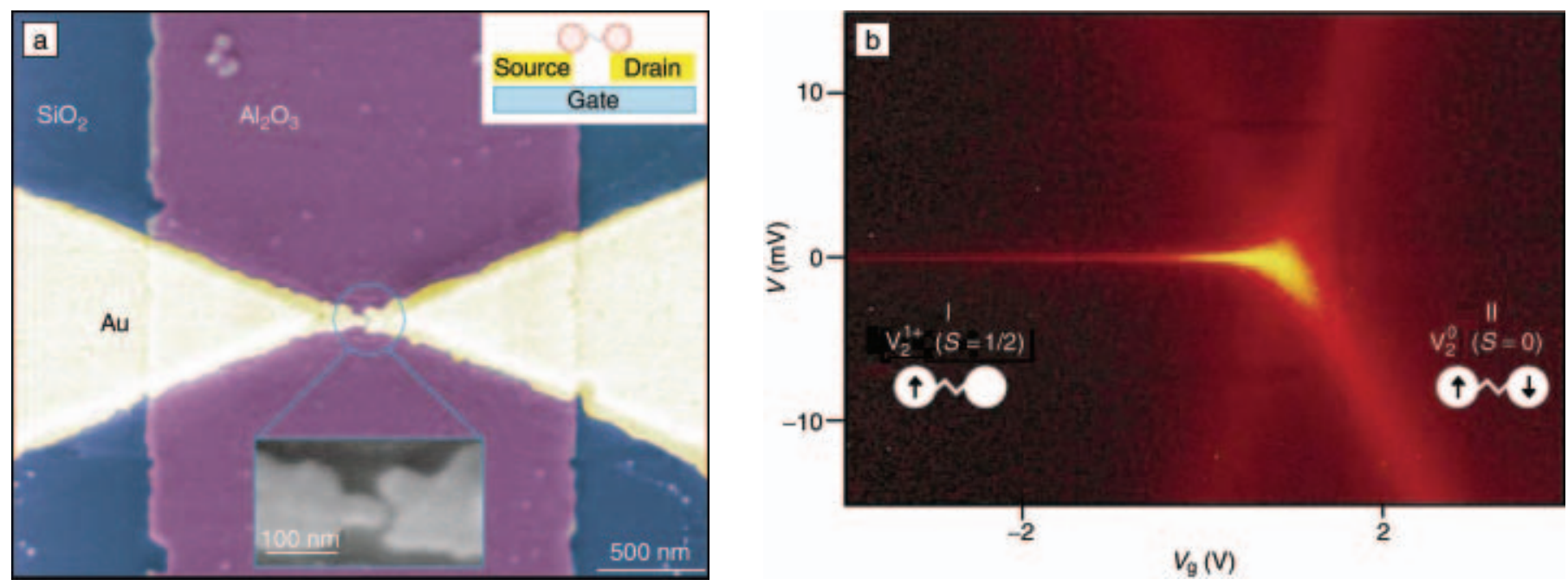

Figure 5. (a) Schematic illustration and enlarged scanning electron micrograph (inset) of a transistor structure (channel length, $\sim 1 \mathrm{~nm}$ ) formed by electromigration of Au electrodes deposited on a $3 \mathrm{~nm} \mathrm{Al} \mathrm{O}_{3}$ gate dielectric with an Al gate. (b) Differential conductance ranges from zero (dark red) to $1.55 \mathrm{e}^{2} / \mathrm{h}$ (bright yellow) as a function of bias and gate voltages. I and II indicate two conductance gap regions, and the diagrams illustrate the corresponding charge and spin states of the divanadium molecule in each region. (Reprinted with permission from Reference 25.) 

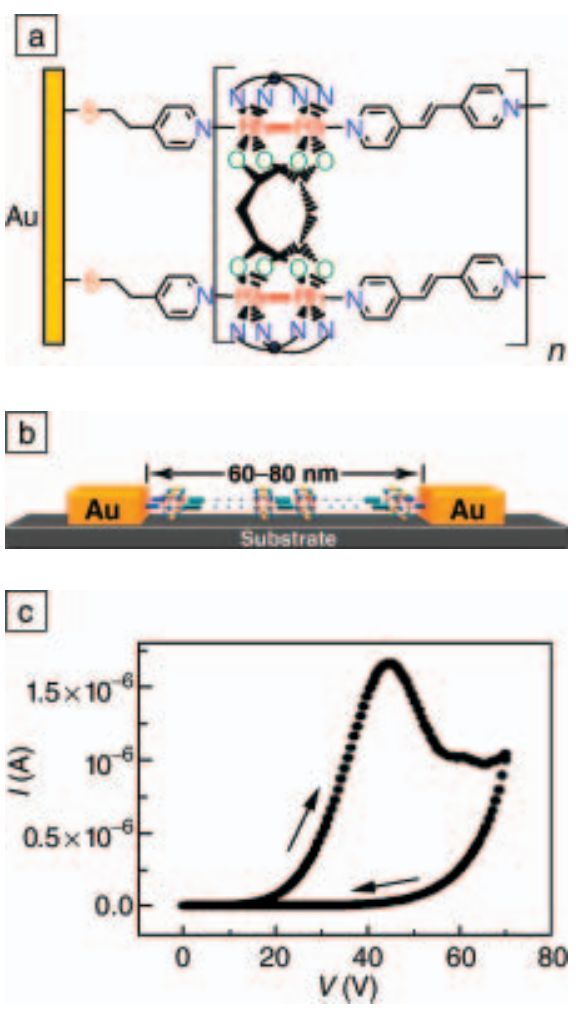

Figure 6. (a) Layer-by-layer assembly of metal-metal bonded coordination compounds templated from source and drain electrodes. (b) Schematic illustration of a metal-metal bonded coordination compound spanning $\mathrm{Au}$ electrodes. (c) Measured I-V

characteristics after the assembly bridges the $\sim 80 \mathrm{~nm}$ channel. Arrows indicate the measured current for the up and down swing in applied voltage. (Reprinted with permission from Reference 26.)

properties. While the science of these systems must come first, molecular and CNT junctions promise intriguing future applications.

\section{References}

1. B. Mann and H. Kuhn, J. Appl. Phys. 42 (1971) p. 4398; E.E. Polymeropoulos and J. Sagiv, J. Chem. Phys. 69 (1978) p. 1836; L. Netzer and J. Sagiv, J. Am. Chem. Soc. 105 (1983) p. 674.

2. A. Leger, J. Klein, M. Belin, and D. Defourne, Thin Solid Films 8 (1971) p. R51.

3. A. Aviram and M.A. Ratner, Chem. Phys. Lett. 29 (1974) p. 277.

4. F.L. Carter, R.E. Siatkowski, and H. Wohltjen, eds., Molecular Electronic Devices: Proc. 3rd Intl. Symp. on Molecular Electronic Devices (NorthHolland, New York, 1989).

5. G. Binning, H. Rohrer, C. Gerber, and E. Weibel, Phys. Rev. Lett. 49 (1982) p. 57.

6. M.C. Petty, M.R. Bryce, and D. Bloor, Introduction to Molecular Electronics (Oxford University Press, Oxford, 1995); C.A. Mirkin and M.A. Ratner, Annu. Rev. Phys. Chem. 43 (1992) p. 719; A. Aviram, ed., Molecular Electronics-Science and Technology (American Institute of Physics, College Park, MD, 1992); A. Aviram, M.A. Ratner, and V. Mujica, eds., Ann. N.Y. Acad. Sci. 852 (1998); J. Jortner and M.A. Ratner, eds., Molecular Electronics (Blackwell Science, Cambridge, MA, 1997); C. Joachim, J.K. Gimzewski, and A. Aviram, Nature 408 (2000) p. 541; M.A. Ratner and M.A. Reed, Encyclopedia of Science and Technology, 3rd ed., Vol. 10 (Academic Press, New York, 2002); V. Mujica and M.A. Ratner, in Handbook of Nanoscience, Engineering and Technology, edited by W.A. Goddard III, D.W. Brenner, S.E. Lyshevshi, and G.J. Iafrate (CRC Press, Boca Raton, FL, 2002); C. Joachim, J.K. Gimzewski, and A. Aviram, Nature 408 (2000) p. 541; P. Hänggi, M. Ratner, and S. Yaliraki, eds., Chem Phys. 281 (2002); M.A. Reed and T. Lee, eds., Molecular Nanoelectronics (American Scientific Publishers, Stevenson Ranch, CA, 2003).

7. C. Joachim, J.K. Gimzewski, R.R. Schlitter, and C. Chavy, Phys. Rev. Lett. 74 (1995) p. 2102. 8. M. Dorogi, J. Gomez, R. Osifchinn, R.P. Andres, and R. Reifenberger, Phys. Rev. B 52 (1995) p. 9071.

9. M.A. Reed, C. Zhou, C.J. Miller, T.P. Burgin, and J.M. Tour, Science 278 (1997) p. 252; H.B. Weber, J. Reichert, F. Weigend, R. Ochs, D. Beckmann, M. Mayor, R. Ahlrichs, and H. von Löhneysen, Chem. Phys. 281 (2002) p. 113; and C. Kergueris, J.P. Bourgoin, S. Palacin, D. Esteve, C. Urbina, M. Magoga, and C. Joachim, Phys. Rev. B 59 (1999) p. 12505.
10. V. Mujica, M. Kemp, and M.A. Ratner, J. Chem. Phys. 101 (1994) p. 6849.

11. S. Datta, Electronic Transport in Mesoscopic Systems (Cambridge University Press, Cambridge, UK, 1995); W. Tian, S. Datta, S.H. Hong, R. Reifenberger, J.I. Henderson, and C.P. Kubiak, J. Chem. Phys. 109 (1998) p. 2874.

12. A. Nitzan, Ann. Rev. Phys. Chem. 52 (2001) p. 681.

13. Y. Xue, S. Datta, and M.A. Ratner, J. Chem. Phys. 115 (2001) p. 4292.

14. D. Mann, A. Javey, J. Kong, Q. Wang, and H.J. Dai, Nano Lett. 3 (2003) p. 1541.

15. A.W. Ghosh, T. Rakshit, and S. Datta, arXiv.org e-print archive, cond-mat/0212166 (accessed March 2004); P.E. Kornilovitch, A.M. Bratkovsky, and M.S. Williams, Phys. Rev. B 66 245413 (2002); A. Troisi and M.A. Ratner, J. Am. Chem. Soc. 124 (2002) p. 14528.

16. E.W. Wong, C.P. Collier, M. Behloradsky, F.M. Raymo, J.F. Stoddart, and J.R. Heath, J. Am. Chem. Soc. 122 (2000) p. 5831.

17. R. McCreery, J. Dieringer, A.O. Solak, B. Snyder, A.M. Nowak, W.R. McGovern, and S. DuVall, J. Am. Chem. Soc. 125 (2003) p. 10748.

18. J. Chen, J. Su, W. Wang, and M.A. Reed, Physica E 16 (2003) p. 17.

19. G.V. Nazin, J.R. Hahn, and W. Ho, Science 302 (2003) p. 77

20. Y. Yaish, J.-Y. Park, S. Rosenblatt, V. Sazonova, M. Brink, and P.L. McEuen, Phys. Rev. Lett. 92046401 (2004).

21. Z.J. Donhauser, B.A. Mantooth, K.F. Kelly, L.A. Bumm, J.D. Monnell, J.J. Stapleton, D.W. Price Jr., A.M. Rawlett, D.L. Allara, J.M. Tour, and P.S. Weiss, Science 292 (2001) p. 2303.

22. G.K. Ramachandran, T.J. Hopson, A.M. Rawlett, L.A. Nagahara, A. Primak, and S.M. Lindsay, Science 300 (2003) p. 1413.

23. J. Reichert, R. Ochs, D. Beckmann, H.B. Weber, M. Mayor, and H.v. Löhneysen, Phys. Rev. Lett. 88 176804-1 (2002).

24. S. Lenfant, C. Krzeminski, C. Delerue, G. Allan, and D. Vuillaume, Nano Lett. 3 (2003) p. 741. 25. J. Park, A.N. Pasupathy, J.I. Goldsmith, C. Chang, Y. Yaish, J.R. Petta, M. Rinkoski, J.P. Sethna, H.D. Abruña, P.L. McEuen, and D.C. Ralph, Nature 417 (2002) p. 722; W. Liang, M.P. Shores, M. Bockrath, J.R. Long, and H. Park, Nature 417 (2002) p. 725.

26. C. Lin and C.R. Kagan, J. Am. Chem. Soc. 125 (2003) p. 336.
Cherie R. Kagan, Guest Editor for this issue of MRS Bulletin, is a research scientist in Physical Sciences at the IBM T.J. Watson Research Center. She has been with IBM since 1998, working on organic and organic-inorganic hybrid materials for thin-film devices. More recently, she has focused her work on molecular assembly and molecular-scale electronics.

Kagan received a BSE degree in materials science and engineering and a BA degree in mathematics from the University of Pennsylvania in 1991. She earned her $\mathrm{PhD}$ degree in electronic materials at the Massachusetts Institute of Technology in 1996. While at MIT, she investigated the optical and optoelectronic prop- erties of nanocrystal assemblies. She then worked as a postdoctoral member of technical staff at Lucent Technologies, Bell Labs, building a scanning confocal Raman microscope to study holographic photopolymers.

Kagan can be reached at the IBM Corporation, 1101 Kitchawan Road, Yorktown Heights, NY 10598, USA; and by e-mail at cheriek@us.ibm.com.

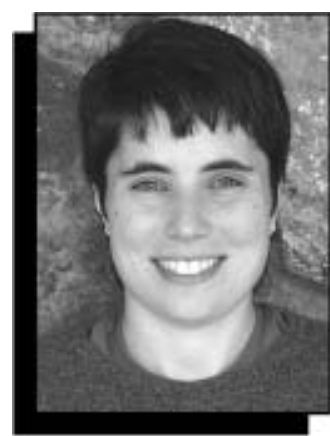

Cherie R. Kagan

Mark A. Ratner, Guest Editor for this issue of

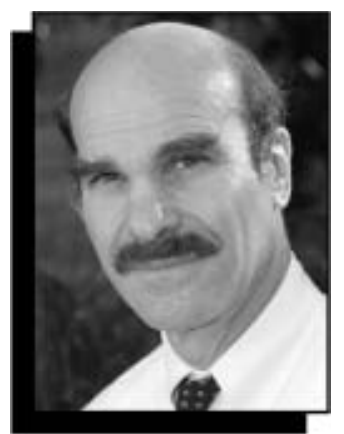

Mark A. Ratner

MRS Bulletin, is a materials chemist and the 


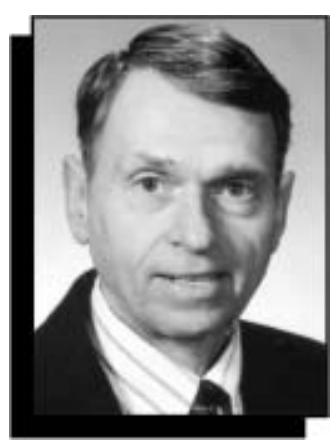

David L. Allara

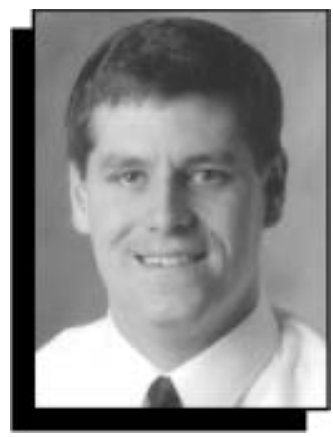

Mark C. Hersam

Morrison Professor of Chemistry at Northwestern University. His work focuses on the interplay between molecular structure and molecular properties, including molecular electronics and optoelectronics, molecular systems design, and biomolecular behavior, as well quantum and classical methodologies for understanding and predicting molecular structure and response. The major focus of his research for the last three decades has been the understanding of charge transfer and charge transport processes based on molecular structures.

Ratner's professional history includes undergraduate work at Harvard University, graduate work at Northwestern, postdoctoral work at the University of Aarhus and the Technical University of Munich, and faculty positions at New York University

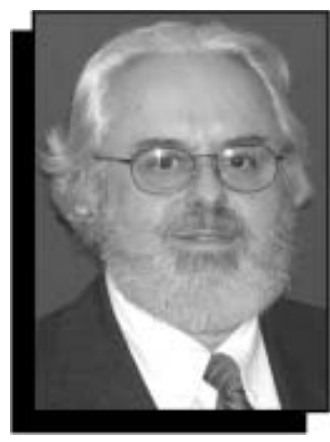

Phaedon Avouris

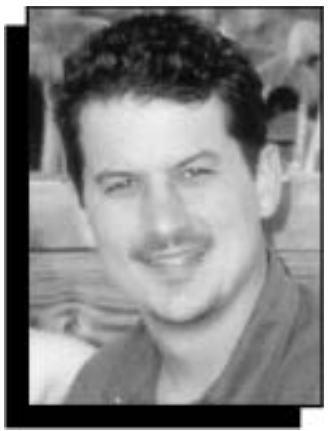

James G. Kushmerick

and Northwestern. He has active international collaborations, particularly in Denmark, Israel, and the Netherlands. He has been awarded the Feynman Prize in Nanotechnology from the Foresight Institute and the Irving Langmuir Award in Chemical Physics from the American Chemical Society and is a member of the National Academy of Sciences, the American Academy of Arts and Sciences, and the International Academy of Quantum Molecular Sciences.

Ratner can reached by e-mail at ratner@chem. northwestern.edu.

David L. Allara is a professor of materials science and chemistry at the Pennsylvania State University. His scientific program is focused on surface chemistry and materials physics involving the preparation, properties, and characterization of molecular

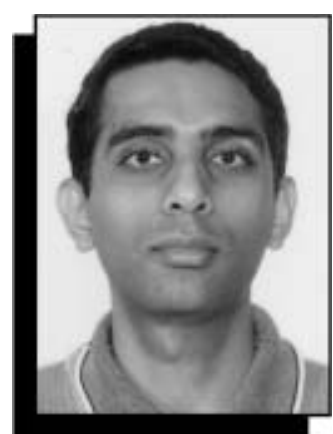

Prashant S. Damle

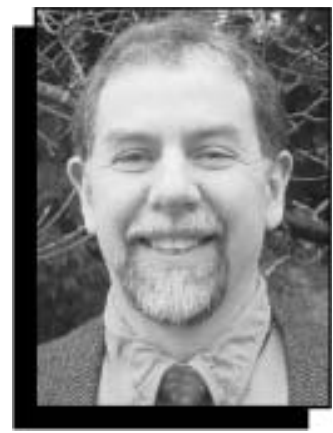

Thomas E. Mallouk

and polymeric thin films, modified surfaces, materials interfaces, and self-assembly of nanomaterials. His current work includes molecular electronics, quantum computing, bio/chem sensors, and biointerfaces.

He received his BS (1959) and PhD (1964) degrees in chemistry (1964) from the University of California at Berkeley and UCLA, respectively. After a 17year career at AT\&T Bell Laboratories, where he was a Distinguished Member of the Technical Staff, he joined the faculty at Penn State in 1987.

He is a AAAS fellow and the recipient of the American Chemical Society Spectroanalytical Chemistry Award (1998), the ACS Adamson Award for distinguished achievements in surface chemistry (2003), and an honorary doctorate of science from Lingköping University, Sweden (2003). His work in molecular

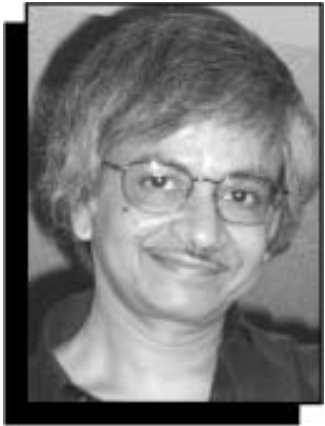

Supriyo Datta

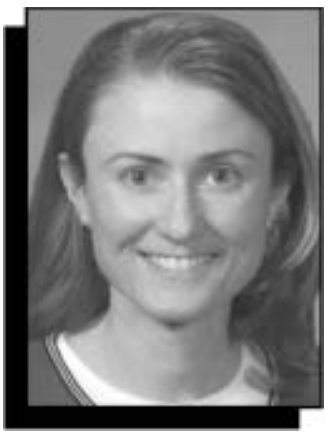

Theresa S. Mayer

self-assembly is widely recognized, with more than 4500 cites for the five most-cited publications.

Allara can be reached by e-mail atdla3@ psu.edu.

Phaedon Avouris is an IBM Fellow and manager of Nanometer-Scale Science and Technology at the IBM T.J. Watson Research Center. He received his BS degree from the Aristotelian University in Greece, and his $\mathrm{PhD}$ degree in physical chemistry from Michigan State University in 1974. After postdoctoral work at UCLA and AT\&T Bell Laboratories, he joined the Research Division of IBM in 1978.

His research interests have ranged from laser studies of fast phenomena to surface physics and chemistry, scanning tunneling microscopy, and atom manipulation. His current research is focused on experimental and theoretical studies of the electrical proper-

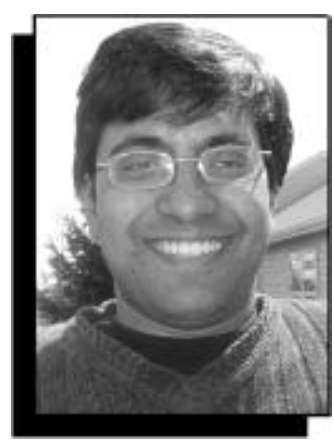

Avik W. Ghosh

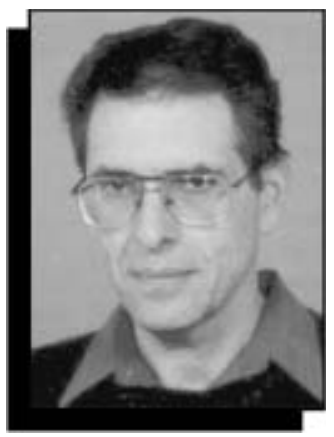

Abraham Nitzan

ties and transport mechanisms of carbon nanotubes, molecules, and other nanostructures. This work includes the design, fabrication, and study of model carbon nanotube and molecular electronic devices and circuits.

Avouris has published 300 scientific papers. He is a fellow of the American Academy of Arts and Sciences, the American Physical Society, the American Association for the Advancement of Science, the American Vacuum Society, and the New York Academy of Sciences. He received the Irving Langmuir Prize from the American Physical Society, the Medard W. Welch

Award from the American Vacuum Society, the Feynman Prize for Molecular Nanotechnology, the ACSIN Nanoscience Prize, the Distinguished Alumnus Award from Michigan State University, and a number of IBM Outstanding Tech- 
nical Achievement awards. He is co-editor of the Springer-Verlag book series on nanoscience and currently serves on the advisory editorial boards of Nano Letters, Nanotechnology, the International Journal of Nanoscience, the Journal of Computational and Theoretical

Nanoscience, Surface Review and Letters, and the Journal of Electron Spectroscopy.

Avouris can be reached by e-mail at avouris@us.ibm.com.

Prashant S. Damle is currently working on the development of $90 \mathrm{~nm}$ Flash memory technology at Intel Corp. in Santa Clara, Calif. He received a BTech degree in electrical engineering in 1997 from the Indian Institute of Technology, Bombay, and $\mathrm{MS}$ and $\mathrm{PhD}$ degrees from Purdue University in 1999 and 2003, respectively. His $\mathrm{PhD}$ research focus was on the modeling of nonequilibrium electron transport in nanoscale electronic devices such as ballistic nano-MOSFETs and atomic and molecular wires.

Damle can be reached by e-mail at damle@ ecn.purdue.edu.

\section{Supriyo Datta is the} Thomas Duncan Distinguished Professor in the School of Electrical and Computer Engineering at Purdue University, where he has been on the faculty since 1981 . His current research interests are centered on the physics of nanostructures, including molecular electronics, nanoscale device physics, spin electronics, and mesoscopic superconductivity.
Datta earned a BTech degree at the Indian Institute of Technology, Kharagpur, in 1975 and his PhD degree from the University of Illinois at Urbana-Champaign in 1979. He received an NSF Presidential Young Investigator Award and an IEEE Centennial Key to the Future Award in 1984, the Frederick Emmons Terman Award from the ASEE in 1994, and shared the SRC Technical Excellence Award (2001) and the IEEE Cledo Brunetti Award (2002) with Mark Lundstrom. He is a fellow of the IEEE, the American Physical Society, and the Institute of Physics and has authored three books, Surface Acoustic Wave Devices, Quantum Phenomena, and Electronic Transport in Mesoscopic Systems.

Datta can be reached by e-mail at datta@ecn. purdue.edu and URL http://dynamo.ecn. purdue.edu/ datta.

Avik W. Ghosh is a research scientist at Purdue University, where he works on electron transport in nanoscale devices, including atomic and molecular wires, nanoscale silicon MOSFETs, hybrid silicon molecular devices, and carbon nanotubes.

Among his other research activities are ultrafast optical

phenomena in semiconductor heterostructures and noise-induced transport in Brownian environments. Ghosh received his MS degree in physics from the Indian Institute of Technology, Kanpur, in 1994 and his $\mathrm{PhD}$ degree in physics from the Ohio State University in 1999, where he received the Ohio State University Presidential
Fellowship. He then joined Purdue University as a postdoctoral research fellow in Electrical Engineering and was appointed to his current position in 2001.

Ghosh can be reached by e-mail at ghosha@ecn. purdue.edu and URL http://dynamo.ecn. purdue.edu/ ghosha.

Mark C. Hersam is an assistant professor in the Department of Materials Science and Engineering at Northwestern University. His research interests include single-molecule devices and sensors, nanofabrication, scanning probe microscopy and spectroscopy, semiconductor surfaces, and carbon nanotubes. He holds a BS degree in electrical engineering from the University of Illinois at Urbana-Champaign; a MPhil degree in microelectronic engineering and semiconductor physics from the University of Cambridge, completed under a British Marshall scholarship; and a $\mathrm{PhD}$ degree in electrical engineering from UIUC, completed with the support of a National Science Foundation Graduate Fellowship and an IBM Distinguished Fellowship.

Before joining Northwestern, Hersam worked for Argonne National Laboratory and the IBM T.J. Watson Research Center, where he performed research on surface acoustic wave sensors and electrical properties of carbon nanotubes, respectively.

Since joining Northwestern in 2000, Hersam has been named an Arnold and Mabel Beckman Young Investigator, a National Science Foundation CAREER
Award recipient, and a Junior Fellow of the Searle Center for Teaching Excellence. He is a member of the American Vacuum Society, the American Physical Society, the American Chemical Society, the Materials Research Society, the American Society of Engineering Education, and the Institute of Electrical and Electronics Engineers.

Hersam can be reached by e-mail atm-hersam@ northwestern.edu and at URL www.hersam-group. northwestern.edu.

James G. Kushmerick is a staff scientist at the U.S. Naval Research Laboratory in the Center for Bio/Molecular Science and Engineering. His current research is focused on nanoscale electronics, encompassing both molecular and bio-organic electronics. Kushmerick earned his $\mathrm{PhD}$ degree in physical chemistry from the Pennsylvania State University. Before taking his current position at the NRL, he spent two years as a postdoctoral fellow at Sandia National Laboratories in the Biomolecular Materials and Interfaces Department.

Kushmerick can be reached by e-mail at kushmerick@nrl.navy.mil.

Thomas E. Mallouk is the DuPont Professor of Materials Chemistry at the Pennsylvania State University. He is best known for his work on inorganic self-assembly and the chemistry of layered, porous, and nanoscale materials. His research has focused on applications of inorganic materials to different chemical problems, in-

cluding nanoscale and molecular electronics, fuel-cell electrochemistry, photochemical energy conversion, chemical sensing, and environmental chemistry. Mallouk received an ScB degree from Brown University. He was a graduate student at the University of California, Berkeley, and a postdoctoral fellow at MIT. In 1985, he joined the chemistry faculty at the University of Texas at Austin, and moved to Penn State in 1993.

Mallouk is the author of about 220 scientific publications and has also edited four books on chemical sensing and solid-state chemistry. $\mathrm{He}$ is currently associate editor of the Journal of the American Chemical Society.

Mallouk can be reached by e-mail at tom@chem.psu.edu.

Theresa S. Mayer is an associate professor in 
Foundation CAREER Award and the Penn State Outstanding Teaching Award. She is currently serving as vice chair of the IEEE Device Research Conference, the Gordon Research Conference on Nanostructure Fabrication, and the UEF Molecular Electronics Conference.

Mayer can be reached by e-mail at tsm2@psu.edu.
Abraham Nitzan has been a professor of chemistry at Tel Aviv University since 1982 and also served as chairman of the School of Chemistry and dean of the Faculty of Science. Nitzan's research is in the field of chemical dynamics and transport phenomena in condensed phases. His recent work has focused on solvation and transport of ions in simple and complex solvents and on electron dynamics and transport at interfaces. Nitzan received his BSc degree in chemistry in 1964, his MSc degree in physical chemistry in 1966 (both from the Hebrew University), and his $\mathrm{PhD}$ degree in 1972 from TAU. He held a postdoctoral Fulbright fellowship at MIT, was a research associate at the University of Chicago, and taught at Northwestern University before joining the faculty at TAU.

He has received the

Kolthoff Prize, the

Humboldt Award, and the Israel Chemical Society Prize. He is a fellow of the American Physical Society and the American Association for the

Advancement of

Science.
Nitzan can be reached by e-mail at nitzan@ post.tau.ac.il.

Ronald G. Reifenberger is a professor of physics at Purdue University. He received his BSc degree from John Carroll University and his $\mathrm{PhD}$ degree in physics from the University of Chicago.

Reifenberger can be reached by e-mail at rr@ physics.purdue.edu.

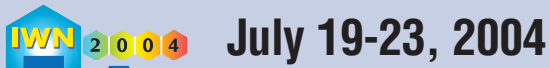

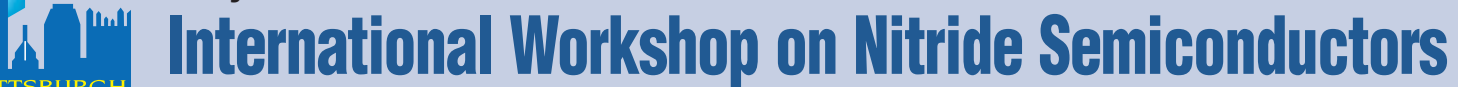 PITTSBURGH
NITRIDE SEMICONDUCTORS Sheraton Station Square • Pittsburgh, Pennsylvania USA}

For the most up-to-date information on this workshop, as well as other meetings and events from the Materials Research Society, visit www.mrs_org/meetings/

\section{Exhibitors... Availability is limited so reserve your booth space today! Contact MRS at 724-779-8312}

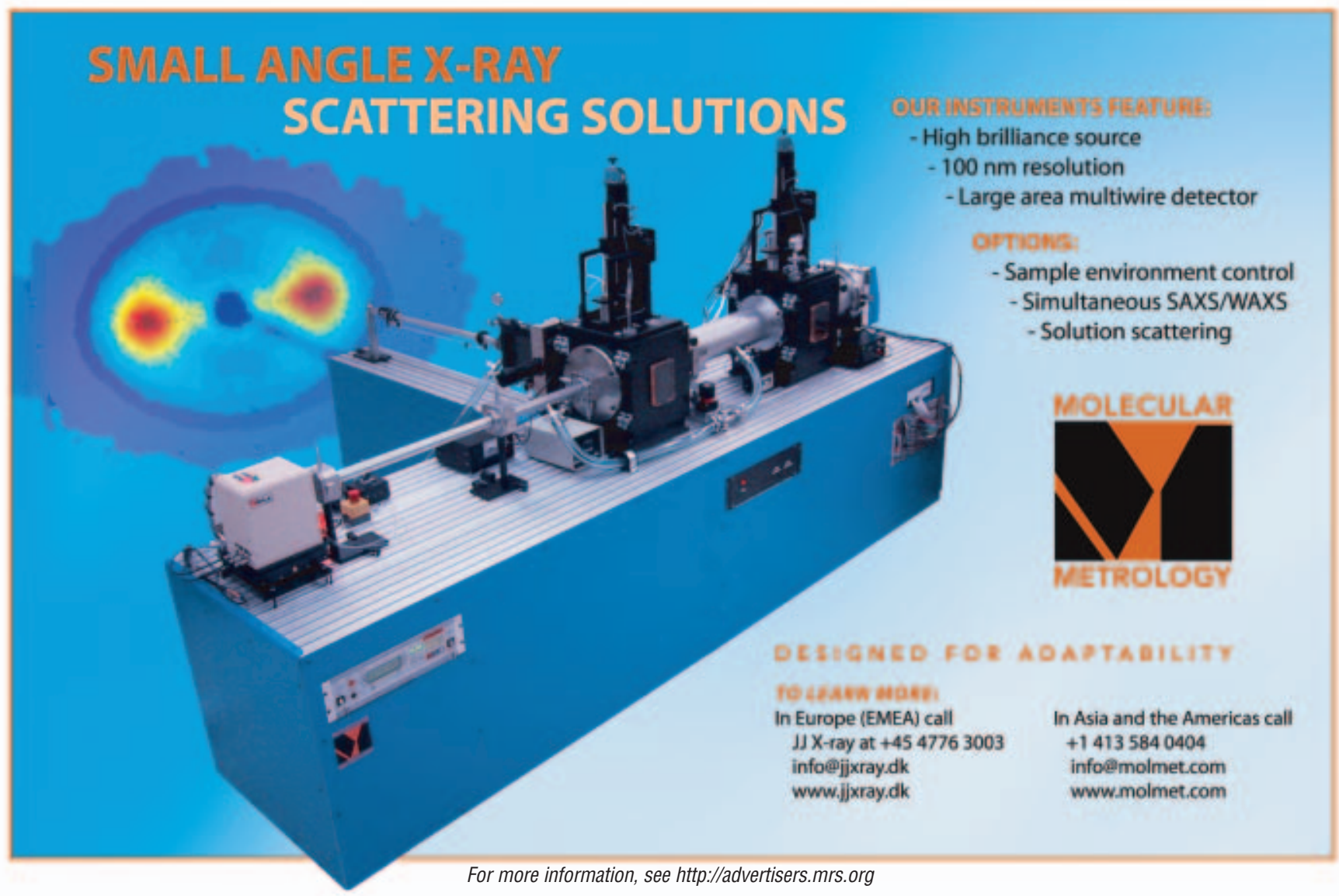

